\title{
Automated chromosome analysis
}

\author{
J.P. Zeidler
}

\section{The increasing interest in and demand for the study of human genetics has led to the introduction on the market of a diverse array of systems for automated metaphase spread location and karyotyping.}

THE defect of a single gene may cause fatal disease, so it is not surprising that the abberation or loss of even a part of a somatic chromosome precludes survival, except in very rare cases. Abnormal cell groupings within the body, particularly leukaemic bone marrow and malignant tumours, may show considerable and characteristic disturbances in their chromosome patterns. The examination and classification (karyotyping) of chromosomes from fetal cells is an essential preliminary to the detection and understanding of congenital disease arising from chromosomal defects. The microscopic examination of chromosomes in the metaphase of cell division is the method widely and routinely used for the detection of chromosomal aberrations in medical genetics, toxicological studies of mutagenicity, the evalution of drug safety and radiation health monitoring.

The tasks involved in locating a number of individual metaphase spreads on a microscope slide, selecting and evaluating the most appropriate spreads, karyotyping and, when required, establishing a karyogram, are very time-consuming. In both routine and research environments the process can become tedious and tiring, however, the importance and consequences of the results of the screening process demand consistency and accuracy from highly trained and qualified staff.

The increasing demands on health services to provide genetic counselling and pregnancy screening, and the burgeoning requirements for mutagenicity testing and radiation health monitoring have added to the pressures on cytogenetic laboratory services. The combined effect of these factors, coupled with the omnipresent need to contain costs, has prompted and accelerated the design and development of instrument systems for automated metaphase spread location and karyotyping.

\section{Early development}

The first instrument for automated chromosome analysis was described in a paper published in 1963 by Robert Ledley'. The instrument consisted of a film digitizer coupled to a computer equipped with software for the extraction of boundaries and the analysis of curvature sequences. The system created considerable interest, and prompted the Medical Research Council (MRC) in the United Kingdom to found a pattern recognition group in its Clinical and Population Cytogenetic Unit in Edinburgh.

The MRC group was initially concerned with designing an instrunient to locate metaphases. The first system was based on a large, custom-built "microscope" with a scanning stage using video imaging, which even early on achieved high search speeds. However, this was at the cost of a large amount of custom hardware, with a resultant lack of flexibility in performance. A system was subsequently developed in conjuction with a team at Tufts New England Medical Center, then led by Peter Neurath. This system was based on a more conventional optical microscope, and used the then new technology of a CCD array scanner ${ }^{2}$.

Growth in the field of cytogenetics prompted several companies which were active in the field of video image analysis to produce adaptations of their existing universal instrument systems by developing specialized software to carry out metaphase spread location and karyotyping, with varying degrees of user interaction. These included Kontron $\mathrm{GmbH}$ in West Germany with the IBAS Video Image Analysis System, Cambridge Instruments in the United Kingdom with the Quantimet 720, and Ernst Leitz Wetzlar GmbH in West Germany, which introduced a metaphase spread and karyotyping program named METFIN in 1980 for their TAS+ System ${ }^{3}$. Around the same time, Joyce-Loebl in the United Kingdom developed an effective system for both metaphase location and karyotyping, based on the MAGISCAN Image Analysis System, in collaboration with John Phillip of the Rigshospitalet in Copenhagen, Denmark, and the Wolfson Image Analysis Unit in Manchester, United Kingdom ${ }^{4}$. A number of companies in the United States have also been active in this field and have enjoyed notable commercial success, including Perceptive Systems, Inc. in Houston, Texas and the Applied Imaging Corporation in Santa Clara, California.

Work continued to progress with the MRC group $^{5-7}$ in Edinburgh in the design and construction of various prototype systems, and in early 1981 a company called Shandon Southern Products Ltd in the United Kingdom commissioned the building of two instrument systems (now marketed by Image Recognition Systems Ltd, Warrington, United Kingdom) which were given the name Cytoscan $110^{8}$.

The availability and relative reduction in the cost of computer memory and framestores has facilitated the development of automated karyotyping, which is available, with a greater or lesser degree of user interaction, from the suppliers of the systems previously described. In addition, systems dedicated to the task of karyotyping have been developed notably one named Karyotec, by the Israeli company Amcor - the first one of which has been installed in the United Kingdom at the Royal Masonic Hospital in London.

\section{On the horizon}

Progressive advances in computer hardware will undoubtedly continue to provide increases in the speed of operation of automated metaphase spread location and karyotyping systems. Today, most instruments have average scanning speeds of under 3-4 minutes per slide. In continuous 24-hour operation a typical instrument can scan a volume of slides that would take an operator using a conventional microscope up to one month to complete, depending on the material and number of karyotypes required.

With such instrument systems now starting to fulfill a central function in cytogenetic laboratories, the demand is emerging for "satellite" microscope systems to enable the cytogeneticist to obtain and interact with the findings of the machine without interrupting or disturbing its routine scanning functions. This requirement has not yet generally been met, but demand for such systems will ensure that they become readily available in the future.

The considerable pressures which led to the development of automated metaphase spread location and karyotyping systems will continue to increase. These, coupled with the increased speed of operation, reliability and convenience of use of the instruments, and their reduction in cost will ensure the deployment and use of such systems on an increasingly wide scale in cytogenetics laboratories.

John P. Zeidler is at E. Leitz Instruments Ltd, 48 Park Street, Luton LU1 3HP, UK. For more information, fill in reader service number 100.

1. Ledley, R.S., Ruddel, F. \& Migeon, B. in Proc. 16th Ann. Conf. Eng. med. Biol., (PTBGME, New York, 1963).

2. Green, D.K. \& Neurath, P.W. J. Histochem. Cytochem. 22, 531 (1974).

3. Reuter, W., Stoll, D. Labor-Praxis in der Medizin 3, (1980) 4. Foster, J. Analyt. quant. Cytol. 7, 192 (1985).

5. Piper, J. \& Rutovitz, D. Pattern Recog. Lett. 4, 397 (1986)

6. Piper, J. \& Rutovitz, D. Pattern Recog. Lett. 3, 119 (1985).

7. Finnon, P., Lloyd, D. C. \& Edwards, A. Mutation Res. 164, 101 (1986)

8. Loerch, T., thesis, Heidelberg Univ. (1986) 